\title{
Research on the Chinese Sport Industry and Related Policy Guided Beneficial Pattern: Theoretical Analysis and Novel Perspective
}

\author{
Yingwei Zhao ${ }^{\text {a, }}{ }^{*}$, Lina Zhu ${ }^{\text {a }}$ \\ Qingdao University of Science \& Technology, Qingdao 261000, China \\ azhaoyingwei66@126.com
}

Keywords: Sport Industry; Beneficial Pattern; Novel Pattern; Financial Policy and Guidance.

\begin{abstract}
With the rapid development of sport science, the management policy and beneficial pattern is urgently needed to be developed. The importance of consumer demand to economic growth is becoming more and more apparent, and sports consumption as an important part of life consumption, not only the change of the consumption structure of the whole, the conversion of consumption patterns, and to promote the development of social productivity and quality enhancement. In this paper, we conduct research on the Chinese sport industry and related policy guided beneficial pattern. We should fully realize that our country sports industry service system is not perfect, the lack of intermediary institutions, poor mobility, fitness entertainment market is not perfect, sport and equipment is not enough, the low level of service guide, product after-sales service to keep up with, the lack of legal and management personnel for the international market and international mediation personnel should be polished. In the future, we plan to do more literature review to modify our proposed pattern.
\end{abstract}

\section{Introduction}

With the continuous development of the socialist market economy in our country, the importance of consumer demand to economic growth is becoming more and more apparent, and sports consumption as an important part of life consumption, not only the change of the consumption structure of the whole, the conversion of consumption patterns, and to promote the development of social productivity and quality enhancement, has the extremely vital significance [1-2]. Therefore, this paper discusses various factors of sports consumption present situation in our country, analysis the advantages and disadvantages, and put forward corresponding countermeasures on the basis of, will play a guidance to the development of future sports consumption and promoting role. Industrial policy which not directly production factors, but the industry development plays an important role. With the development of our country sports industry, sports industry policy choice has become an inevitable in the sports industry policy related research started in the $1990 \mathrm{~s}$, some scholars firstly explains the basic concept of the sports industry policy, to study from the perspective of macro policy of sports industry. Many scholars have in common is the sports industry policy is one of the national economic policy, as an economic lever to intervene and control the advantages of developing sports industry. Based on the research of the existing sports industry policy, it is concluded that the main problems fall into five categories: the connotation of sports industry policy and classification system research, various kinds of sports industry policy study and use for reference foreign sports industry policy, industry policy, mechanism of action research and rational industrial policy research. In sports industry policy research in recent years, gradually incline to phase quantitative analysis, further elaborated the sports industry policy selection or optimization [3].

According to the extrinsic manifestation of sports consumption, it can be divided into sightseeing, participative sports consumption and sports consumption traditional sports consumption [4]. So-called ornamental type sports consumption, it is to point to watch all kinds of sports, performances, exhibitions and other of consumption. This kind of consumption under the condition of material life level rising, people will go to pursue, belongs to a higher level of spiritual enjoyment. Joining in sports consumption refers to for entertainment, fitness and take part in different kinds of 
sports, fitness training, sports consumption of health care, etc. With the increasing of people's leisure time, in pursuit of a higher quality of life, will more actively into the practice of sports, as a result, this kind of sports consumption has great potential for market development [5]. Traditional sports consumption refers to the individual to buy sports clothing shoes and hats and sports equipment and other sports items of consumer spending. With the expansion of our country sports socialization and sports population increase, traditional sports consumption will be rapid growth. For now, China's consumer spending form is given priority to with traditional sports consumption. Therefore, in this paper, we conduct research on the Chinese sport industry and related policy guided beneficial pattern. Our point of view is insightful and important. The detailed discussion will be introduced in the following parts.

\section{Our Methodology and Research}

The Influence Factors of Sports Consumption. Growth of residents' sports consumption in our country, and the overall level of economic development and economic factors such as personal income and consumption levels are closely related, but also by the residents' consumption psychology, consumption habits, leisure time, factors such as social cultural background, the sports level of comprehensive effect. Engels divided the human needs for survival needs which enjoy the needs and development needs three levels. Sports consumption clearly belongs to the scope of demand and developmental demand, because of its greater elasticity of demand and therefore, in the case of the low level of income, the demand for sports consumption will not have a rapid increase. And that is basically used in such as sportswear, sports shoes and socks and simple sports equipment and material consumption, such as for sports labor service consumption expenditure proportion is very low, only a small number of people in the sports participants have to profit-making stadiums for the sports consumption. And, in the sports physical consumption market, cheap price of sports goods is the first choice for most consumers to use. Although at present our country sporting goods market, there is a different class, different price of sports goods, from a few yuan to hundreds of yuan, even thousands of yuan price of everything, make consumers for a variety of sports goods faced with the choice of diversification. However, the vast majority of sports consumers purchasing power, to accept and use only cheap price of sports goods, while for those high grade and high value few takers or away. Entering the 21st century, with the continuous development of our country's economy, the income level of people has improved considerably. Even by purchasing power parity terms, China's per capita consumption level is low. According to Engel's law, a family income is less, its income used to buy food spending accounts for the greater the ratio, with the increase of residents' income, the used in the proportion of food expenditure in total expenditure will decline, and used for cultural entertainment and sports consumption spending will rise gradually. Visible, residents in our country's sports consumption level is very low at present, the root causes is determined by the state of the economy in our country, and won't have too big growth in the short run. In the low level of current sports services, meet the demand of the people's growing sports consumption and the larger gap. For example, in the domestic and domestic basketball level has a lot to do to improve. In sports should also be stressed that "strives for the survival by the quality". At home near a lot of sports venues, but there is no swimming pool and sauna room. When a large sports consumers just out of the sports venues, the mood how urgent for bathing places. Therefore, aiming at the fact that sports consumption level is not ideal, we not only from the outside to find the reason, also should be based itself on at the same time, many from improve the service quality fluctuation exercise. Therefore, there are some existing influence factors of sports consumption.

The Allocation of Resources Measurement Model. Government through industrial policy means unbalanced allocation of different industries, promoting and inhibiting industry exhibition, and eventually promote industrial total priority to the development and growth. In the allocation of resources and advanced degrees as indicators of evaluation of the effect of sports industry policies, lots of parameters are taken into consideration. Because of the sports industry policy is different, make incremental differences between the allocation of resources, sports industry characterized by 
incremental resources development of the industry at a faster rate than or less than the average level of development, the slope is the allocation of resources. The inputs of gradient calculation formula are expressed in the expression 1.

$$
S_{i}^{k}=k_{i}^{\prime} / \bar{K}^{\prime}-1 \quad(i=1,2,3, \ldots, n)
$$

Advance the development of the industry in the market economy of unbalanced growth in a priority position, advanced degrees, and the higher growth will be the priority status, but it is a state of relative. In the development of sports and related industry to the national sports industry is average growth rate of the reference standard. The formula 2 shows the calculation process.

$$
e_{i}=s_{i}-\bar{S} \quad(i=1,2,3, \ldots, n) \quad s_{i}>0, \bar{S}>0
$$

With the aid of mathematical models in economics, from existing in the sports industry statistics maximum absorbing useful information, to judge the difference degree and regional industrial policy in China. Sports industry policy implementation, using quantitative measure economic data have certain scientific, but there are other influencing factors, in this study, under the assumption that is not affected by other factors of standardized computing. The inclination of the resource allocation effect is expressed as the formula 3 and 4.

$$
\begin{aligned}
& \bar{K}_{t}^{\prime}=\left(C_{t}-C_{t-1}\right) / C_{t-1} \\
& S_{d, t}^{k}=k_{d, t}^{\prime} / \bar{K}_{t}^{\prime}-1
\end{aligned}
$$

Resource allocation of advanced degrees is not simply industrial output growth velocity and the average output of the national economy growth is presupposed. To get the name of the $x$ industry added value and actual value, you must eliminate price factors that is the rate of inflation, the consumer price index (CPI) are used to indicate. Sports consumption is an aspect of public life, it is in people's material life conditions under the premise of meet the basic life to need, in order to pursue the development of individual and enjoy of make a choice to adapt to the higher level, is a kind of sports functions on the basis of the perception of new types of consumption. Sports consumption as an important part of modern life consumption, to a certain extent reflects a country's national quality, education level and civilization degree.

Suggestions and Solutions for the Current Policy. With the continuous development of productivity and the increase in the general standard of residents' income level, value pursuit must also presents the diversification, this needs us to guide and take effective measures to correct consumption in sports consumption demand growth. Sports power resource allocation: here refers to sports power resources show the structure of the power structure, sports power resource allocation imbalance is mainly refers to as a result of the unbalance of system arrangement pattern defect caused by the interests, there are two: one is the offside of the management, the administrative department of sports is difficult to give up their own interests, the administrative department to administrative control measures interference in the professional league development, moral self-discipline mechanism industry collapse, the healthy development of sports industry has hampered. Football professionalization development in our country, for example, football sports management center to participate in the international and domestic competition for the lever controls the matches actually, make football professional road difficult to promote reasonable, more to the identity of the system is arranged around the earnings and the distribution of the football league. Visible, all stakeholders power resource allocation imbalance has or not is the main reason for the development of football professionalization. First of all, it must develop a reasonable price. Reasonable price set by means of sports consumption price in accordance with the actual consumption level of urban and rural residents in China. According to the degree of different income levels, different culture, different areas to segmentation, target market for different market with different sports services; Reasonable price means the consumer psychological expectations of sports consumer goods price and the actual price set by the gap between the rationalization; Reasonable price means by improve the quality of sports services, make consumers in the consumer feel content is worth somewhat, satisfaction degree is higher. Second, must make full use of mass media, increase publicity of sports. The influence of 
media on sports has been there for all to see. So, we should give full play to the guiding role of mass media, enhance the awareness of sports, at the same time, the media itself is a carrier of the people in sports consumption, through the medium of the masses, make people understanding of sports greatly enhanced. Third, promote the common sports consumption, efforts to expand the sports population. Sports consumption of civilian is the necessity of market economy, is the objective requirement of the sports market development, the increase in consumption. Therefore, we should widely in some towns, community construction has a comprehensive sports facilities in place, make broad residents have more participation opportunity, at the same time to strengthen the construction of sports instructor team, make full use of community coaching center, clubs and other ways to meet different consumer needs, and increase the popularity of sports science and technology, education and promotion. Through the above measures, and constantly expand the sports population and improve the number of sports consumers. Fourth, increase the sports consumption market services. Must strive to increase the service contents, improve service levels, which truly let consumer satisfaction. The corresponding suggestions will enhance the current policy.

\section{Summary and Conclusion}

With the continuous development of the socialist market economy in our country, the importance of consumer demand to economic growth is becoming more and more apparent, and sports consumption as an important part of life consumption, not only the change of the consumption structure of the whole, the conversion of consumption patterns, and to promote the development of social productivity and quality enhancement, has the extremely vital significance. On the basis of sports statistics and related industries in China, using the method of econometrics to input and output value system as the analysis framework, concrete slope through the allocation of resources with advanced degrees respectively to evaluate our country sports industry policy tilt and degree of the advanced development of industrial policy implementation. This paper introduces the novel pattern for the policy guidance, which will enhance the current situation. We should fully realize that our country sports industry service system is not perfect, the lack of intermediary institutions, poor mobility, fitness entertainment market is not perfect, sport and equipment is not enough, the low level of service guide, product after-sales service to keep up with, the lack of legal and management personnel for the international market and international mediation personnel, etc., and as members of the WTO and opening their sports service market, improve the international competitiveness of sports service there is a larger gap. In the future, we plan to do more related research on the state-of-the-art other patterns to polish the present model.

\section{References}

[1] Cheng-yun Z. An Analysis of Olympic Plate of A-share Listed Company Promoting Chinese Sport Industry [J]. Journal of Beijing Sport University, 2009.

[2] Yadong C. Influence of Adidas Merger Reebok on Chinese Sport Goods Industry [J]. China Market, 2008.

[3] Liangju W. Academic Opinion of Promoting the Chinese Sport Industry to the Primary Industry of National Economy [J]. Modern Finance and Economics (Journal of Tianjin University of Finance and Economics), 2009.

[4] Fang-lin D. On the Influence of Shanghai World Expo on Chinese Sport Industry Development [J]. Journal of Inner Mongolia Finance and Economics College, 2012.

[5] Ying-chun Z, Chang-zheng W. Countermeasures to Advance the Development of Chinese Sports Industry [J]. Journal of Shenyang Sport University, 2006. 